\title{
Modeling Cable-Driven Joint Dynamics and Friction: a Bond-Graph Approach
}

\author{
Daniele Ludovico $^{1,2}$, Paolo Guardiani ${ }^{1,2}$, Alessandro Pistone ${ }^{2}$, Jinoh Lee ${ }^{3}$, \\ Ferdinando Cannella ${ }^{2}$, Darwin G. Caldwell ${ }^{2}$ and Carlo Canali ${ }^{2}$
}

\begin{abstract}
Cable-driven joints proved to be an effective solution in a wide variety of applications ranging from medical to industrial fields where light structures, interaction with unstructured and constrained environments and precise motion are required. These requirements are achieved by moving the actuators from joints to the robot chassis. Despite these positive properties a cable-driven robotic arm requires a complex cable routing within the entire structure to transmit motion to all joints. The main effect of this routing is a friction phenomenon which reduces the accuracy of the motion of the robotic device. In this paper a bond-graph approach is presented to model a family of cable-driven joints including a novel friction model that can be easily implemented into a control algorithm to compensate the friction forces induced by the rope sliding into bushings.
\end{abstract}

\section{INTRODUCTION}

The most interesting characteristic of a cable-driven mechanism is the option to move actuators from joints to chassis reducing the overall weight of the moving structure and increasing the payload capability of the robot. This property makes possible to assemble several joints together into a hyper-redundant manipulator able to move into wide, but constrained environments. Furthermore, the presence of cables introduces compliance in motion transmission increasing the safety of interaction with unstructured environments. All these attributes promote the cable-driven mechanism to widespread in many applications. In medical field, where accurate motion, dexterity and safe interaction are crucial aspects, the cable-driven mechanism is adopted to realise system for minimally invasive surgery [1]. In industrial fields, cable-driven mechanisms appear in various application ranging from a parallel robot for logistics [2] to hyper-redundant robots for the inspection of complex and constrained devices [3]-[5] or maintenance in harsh environment [6]-[8] that cannot be reached by humans. In bio-inspired robots cable driven mechanism are often used to mimic limb tendons [9].

One of the drawback of such mechanisms is the necessity to route the cables all along the structure complicating the design and introducing friction due to their interaction with bushing and pulleys. Requirements on motion accuracy lead

\footnotetext{
1 Authors are with Department of Informatics, Bioengineering, Robotics and System Engineering, University of Genoa, 16126, GE, Genoa daniele.ludovicodit.it

2 Authors are with Advanced Robotics Department, Istituto Italiano di Tecnologia (IIT), 16163, GE, Genoa, Italy

$3 \mathrm{~J}$. Lee is currently with the Institute of Robotics and Mechatronics Center, German Aerospace Center (DLR), 82234 Weßling, Germany. He was with the Advanced Robotics Department, Istituto Italiano di Tecnologia (IIT), 16163 Genoa, Italy
}

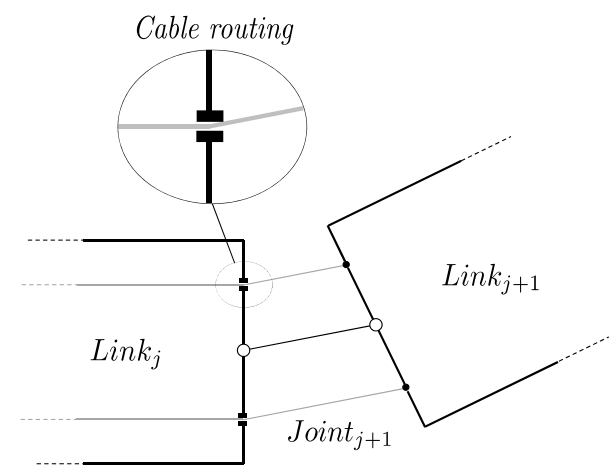

Fig. 1. Schematic of the cable-driven joint family considered in this work.

to define a precise model for cable-driven mechanism which considers also friction phenomena.

This work focuses on modeling a family of cable-driven joints in which the cable routing along the structure has been fulfilled through bushings as shown in Fig 1. The bondgraph approach [10], a graphical modeling technique, has been selected to determine the dynamic equations of the mechanism. Even if the bond-graph features fit perfectly with cable-driven mechanisms there are few examples of such mechanisms modeled through bond-graph [11]. This approach allows to separately analyse the different parts constituting the mechanism to better understand the phenomena involved in each subsystem. A complete simulation of the whole system can be obtained connecting together each subsystem through power bonds. In this modeling framework it is easy to understand how the friction forces act and consequently how to include them in the dynamic equations.

Friction is one of classic, yet challenging problems in practical system modeling. Several friction models have been defined in literature: static friction model consists in a combination of Coulomb, viscous, stiction and Stribeck terms, and an accurate description of friction is given by dynamic models such as Dahl or LuGree friction model [12]. For cable-driven mechanisms, friction forces produced by pulleys can be modeled as a function of the cable tension, velocity, wrap angle and number of pulleys [13]. An attempt to define a general formulation for multi-node sliding cable is presented in [14]. To describe the friction force produced by sliding rope into bushing a modified version of the Dahl's model has been proposed [15]. However, aforementioned models are not able to correctly describe the friction forces generated by cables sliding into bushings or are too complex 


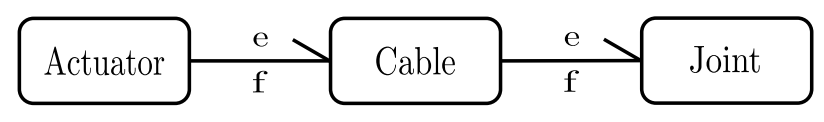

Fig. 2. General bond graph representation of a cable driven joints.

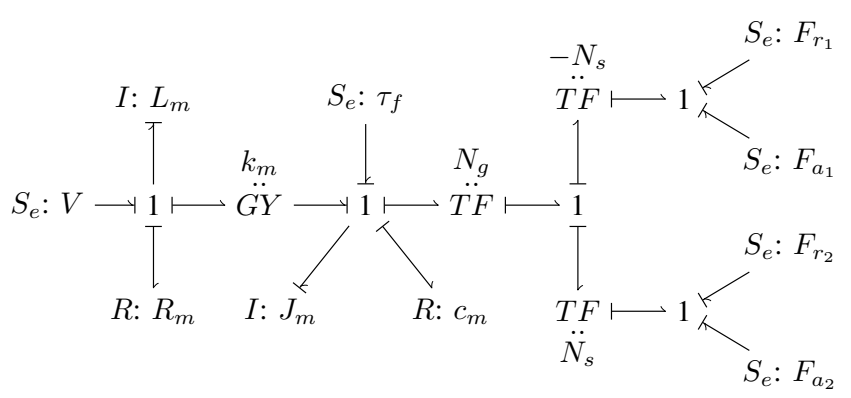

Fig. 3. Bond graph describing the linear actuator model.

to be easily implemented in a real time controller.

In this paper a novel method to model the friction of a cable sliding into a bushing is proposed; the aim is to define a simple model that can accurately describe the friction forces of sliding cable to eventually improve the performances of a real time control algorithm for cable-driven mechanism. An hardware prototype has been used to validate the proposed model. The remainder of this paper is organised as follows: in Section II the bond-graph modeling approach for cabledriven joints is presented and the dynamic equations of the system are derived. In Section III the mathematical friction model for cables sliding into bushing is presented. Section IV presents the hardware setup and the experimental tests performed to identify the sliding rope friction coefficients, and the simulation results are compared with the real device behaviour. In Section V the conclusion is drawn and future works are listed.

\section{Bond-GRAPH Modeling OF CABLE DRIVEN MeChANISM}

A cable-driven joint can be seen as the connection of three macro subsystems: a certain number of actuators, several cables and a joint mechanism. The physical connection among them can be represented in terms of generalised efforts $(e)$ and flows $(f)$ as illustrated in Fig. 2.

In this scenario the bond-graph approach allows to define a model for each of these subsystems separately making easier the understanding of the main phenomena involved in all these parts isolating them to define identification procedures to estimate uncertain parameters such as friction or stiffness. Furthermore this technique makes it easy to substitute different models of the same subsystem so as to favor the accuracy or the speed of the simulation. From the graphical representation of bond-graphs the dynamic equations of each subsystem can be derived where the state variables are represented by generalized momenta and



Fig. 4. Bond graph describing the cable model.

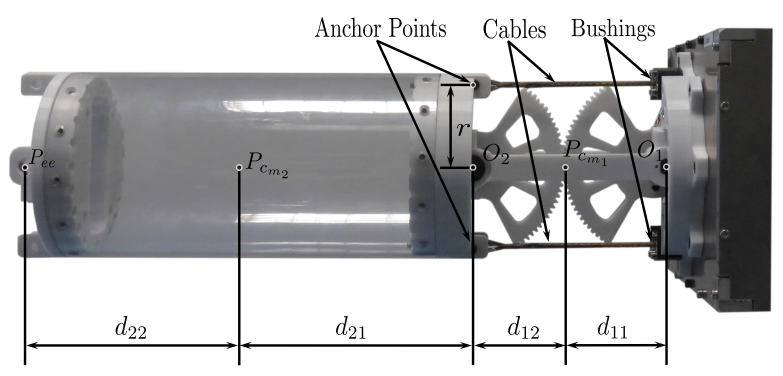

Fig. 5. Cable driven joint tested during the experiments.

displacements. Finally each subsystem can be connected to each other to obtain a complete simulation of the mechanism. In this work a real cable-driven joint has been considered to validate the proposed model. The following subsections present the bond-graph model for each subsystem of the selected cable-driven joint.

\section{A. Actuator Model}

The linear actuator under test is made by a geared brushless DC motor that moves two parallel ball nuts at the same speed but in opposite direction. From the bond-graph illustrated in Fig. 3, it is possible to derive the state space representation of the linear actuator as

$$
\begin{aligned}
& \dot{x}_{1}=-\frac{R_{m}}{L_{m}} x_{1}-\frac{k_{m}}{J_{m}} x_{2}+V, \\
& \dot{x}_{2}=\frac{k_{m}}{L_{m}} x_{1}-\frac{c_{m}}{J_{m}} x_{2}+\tau_{f}+N_{g} N_{s}\left(\Delta F_{r}+\Delta F_{a}\right), \\
& \dot{x}_{3}=x_{2},
\end{aligned}
$$

where $x_{1}, x_{2}$ and $x_{3}$ are the state variable representing respectively the flux linkage, the angular momentum and the integral of the angular momentum of the motor, $V$ represents the voltage applied to the motor, $\tau_{f}$ is the gear friction torque, which can be experimentally determined trough an identification procedure described in Section IV, $\Delta F_{r}=F_{r_{1}}-F_{r_{2}}$ is the force applied by the cables and $\Delta F_{a}=F_{a_{1}}-F_{a_{2}}$ is the friction force produced by the sliding cables, which is described in Section III. The actuator parameters are listed in Table I.

\section{B. Cable Model}

The cables selected in this work are made by a synthetic fibre called Zylon. While this material shows a non linear behaviour for low tension, under the assumption that the preload is sufficiently high, it is possible to approximate the cable model with a parallel between a linear spring and a damper. Fig. 4 illustrates the equivalent bond-graph representation of this model. The resulting state space description 


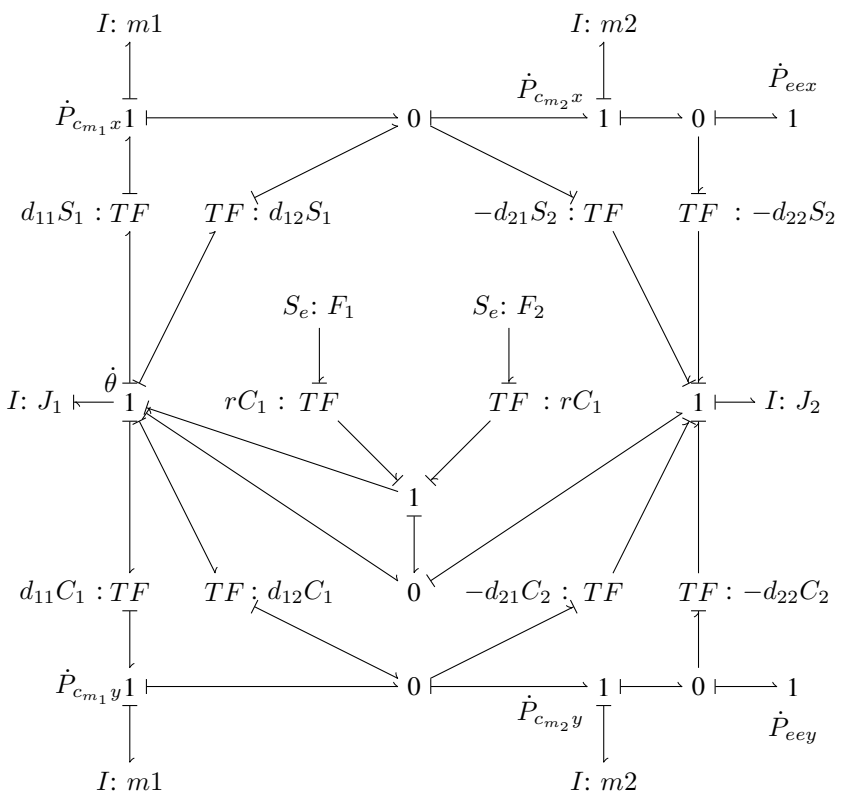

Fig. 6. Bond graph describing the joint model.

is defined by

$$
\dot{x}_{4}=v_{j}-v_{a}
$$

where $x_{4}$ is the state variable representing the elongation of the cable, $v_{j}$ and $v_{a}$ are linear velocity of the joint and actuator, respectively. From the state equation (2) the forces applied by each cable can be computed as

$$
F_{r}=K x_{4}+c\left(v_{j}-v_{a}\right)
$$

where the stiffness $K$ and internal damping $c$ can be estimated through an identification procedure described in Section IV.

\section{Joint Model}

The selected cable-driven mechanism consists of two rigid links and two parallel revolute joints whose rotation angles are constrained to be same through a gear system. Fig. 5 shows the joint prototype, where $O_{1}$ and $O_{2}$ represent respectively the first and second rotation axes, and $P_{c_{m_{1}}}$ and $P_{c_{m_{2}}}$ are the center of mass $(\mathrm{CoM})$ of the first and second links, respectively, while $P_{e e}$ is the end effector position. $P_{c_{m_{1}}}, P_{c_{m_{2}}}$ and $P_{e e}$ are described by

$$
\begin{aligned}
P_{c_{m_{1}}} & =\left[\begin{array}{l}
d_{11} \cos (\theta) \\
d_{11} \sin (\theta)
\end{array}\right], \\
P_{c_{m_{2}}} & =\left[\begin{array}{l}
l_{1} \cos (\theta)+d_{21} \cos (2 \theta) \\
l_{1} \sin (\theta)+d_{21} \sin (2 \theta)
\end{array}\right], \\
P_{e e} & =\left[\begin{array}{l}
l_{1} \cos (\theta)+l_{2} \cos (2 \theta) \\
l_{1} \sin (\theta)+l_{2} \sin (2 \theta)
\end{array}\right],
\end{aligned}
$$

where $\theta$ denotes the angular position of the joint, $d_{11}$ and $d_{12}$ represent the distance between the CoM of the first

\begin{tabular}{|c|c|c|c|}
\hline Symbol & Value & Unit & Description \\
\hline \multicolumn{4}{|c|}{ Actuator Parameters } \\
\hline$L_{m}$ & 0.471 & $m H$ & Motor inductance \\
\hline$R_{m}$ & 0.444 & $\Omega$ & Motor resistance \\
\hline$c_{m}$ & 10.319 & $m N m / s$ & Motor viscous friction \\
\hline$J_{m}$ & 349.88 & $\mathrm{gcm}^{2}$ & Total inertia \\
\hline$k_{m}$ & 102.4 & $m N m / A$ & Motor torque constant \\
\hline$N_{\text {gear }}$ & $1: 80$ & & Gear reduction ratio \\
\hline$N_{\text {screw }}$ & 5 & $m m$ & Screw pitch \\
\hline \multicolumn{4}{|c|}{ Joints Parameters } \\
\hline$m_{1}$ & 0.217 & $k g$ & $1^{\text {st }}$ link mass \\
\hline$J_{1}$ & 0.0007 & $\mathrm{kgm}^{2}$ & $1^{\text {st }}$ link inertia \\
\hline$m_{2}$ & 0.806 & $\mathrm{~kg}$ & $2^{\text {nd }}$ link mass \\
\hline$J_{2}$ & 0.00016 & $\mathrm{kgm}^{2}$ & $2^{\text {nd }}$ link inertia \\
\hline$d_{11}$ & 47 & $m m$ & $\bar{O}_{1} P_{c_{m_{1}}}$ \\
\hline$d_{12}$ & 103 & $m m$ & $\bar{O}_{2} P_{c_{m_{1}}}$ \\
\hline$d_{21}$ & 12 & $m m$ & $\overline{\mathrm{O}}_{2} P_{c_{m_{2}}}$ \\
\hline$d_{22}$ & 338 & $m m$ & $\bar{P}_{e e} P_{c_{m_{1}}}$ \\
\hline$r$ & 65 & $\mathrm{~mm}$ & Anchor point distance \\
\hline
\end{tabular}
link and $O_{1}$ and $O_{2}$, respectively, $d_{21}$ and $d_{22}$ represent the distance between the CoM of the second link and $\mathrm{O}_{2}$ and
TABLE I

ACTUATOR PARAMETERS NOMINAL VALUES

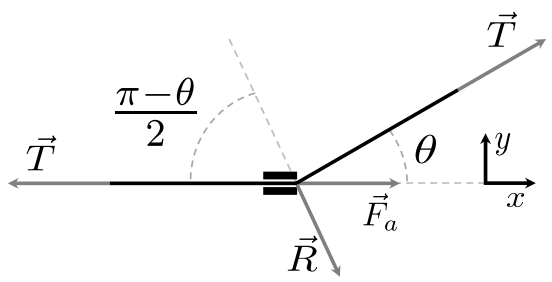

Fig. 7. Schematic of the forces acting on a sliding cable.

$P_{e e}$, respectively, and $l_{1}=d_{11}+d_{12}$ and $l_{2}=d_{21}+d_{22}$ represent the length of the first and second links.

The bond-graph depicted in Fig. 6 is obtained through (4). This gives the following dynamic equation:

$$
\dot{x}_{5}=\frac{\frac{-4 m_{2} l_{1} d_{21} \sin (\theta)}{J_{1}^{2}} x_{5}^{2}+r \cos (\theta) \Delta F_{r}+\tau_{g}}{1+\frac{4 J_{2}+m_{1} d_{11}^{2}+m_{2}\left(l_{1}^{2}+4 l_{1} d_{21} \cos (\theta)+4 d_{21}^{2}\right)}{J_{1}}},
$$

where $x_{5}$ denotes the state variable representing the angular momentum of the joint, $m_{1}, J_{1}$, and $m_{2}, J_{2}$ are respectively the mass and inertia of the first and second links, $r$ is the distance of the cable anchor point from the center of the flange and $\tau_{g}$ is the torque due to gravity which is described as

$$
\tau_{g}=d_{11} \cos (\theta) F_{g_{1}}+\left(l_{1} \cos (\theta)+2 d_{21} \cos (2 \theta)\right) F_{g_{2}},
$$

where $F_{g_{1}}$ and $F_{g_{2}}$ are the gravity forces acting on the first and second links respectively. Table I shows the values of the joint parameters.

\section{Sliding CABle Friction Model}

In this section the mathematical friction model of the cable sliding into a bushing is presented. The aim is to define a simple model that can be easily inserted as feedforward term in a control scheme to improve the trajectory tracking performances of cable-driven mechanisms. Under the assumption 


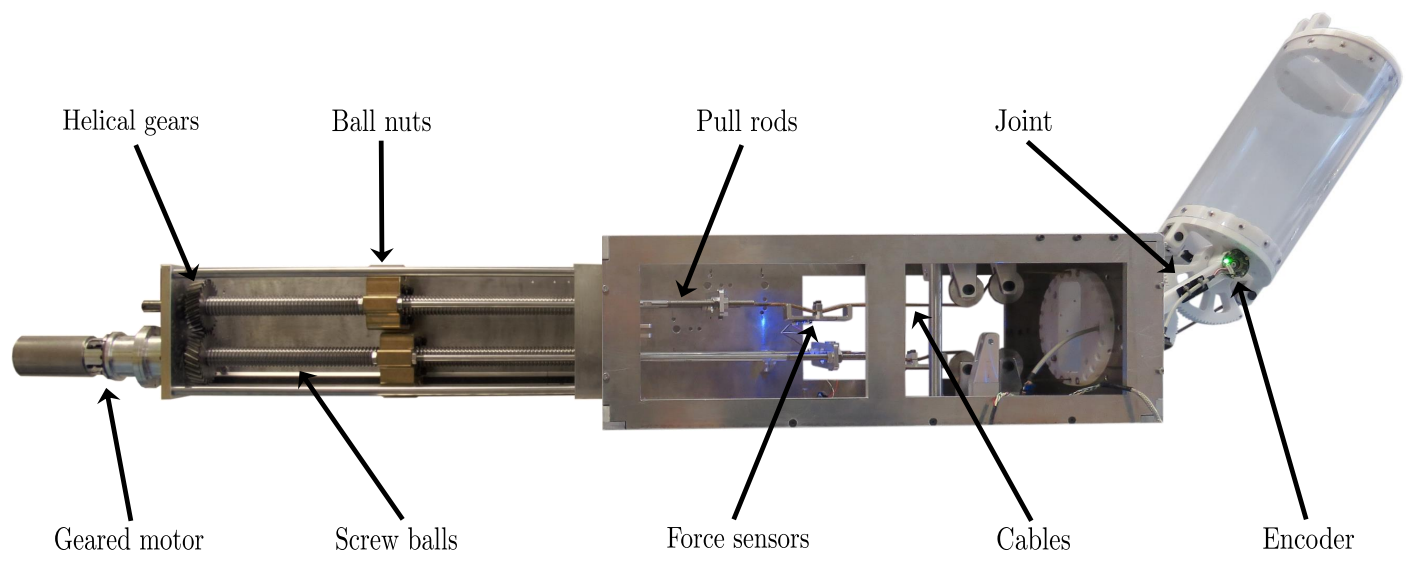

Fig. 8. Hardware setup for the experiments with the prototype of the cable-driven joint and its actuation system.

that the relative velocity between cable and bushing is low it is possible to neglect viscous friction phenomena and consider only stiction. Then the static friction acting on the cable can be computed as

$$
F_{a}=\mu|\vec{N}|
$$

where $\mu$ represents the static friction coefficient and $N$ is the force normal to the contact point. Fig. 7 shows a schematic of the forces acting on the cable in a given configuration $\theta$. From the equilibrium equation it is possible to find the reaction force $\vec{R}$ given by the bushing as follows:

$$
\vec{R}=\left[\begin{array}{c}
T(1-\cos (\theta)) \\
-T \sin (\theta)
\end{array}\right]
$$

where $T$ is the norm of the preload applied to the cable. Considering that $\vec{N}$ lies on the bisector of the angle between the entrance and exit direction of the cable the module of $\vec{N}$ can be computed as the projection of $\vec{R}$ on such bisector

$$
|\vec{N}|=2 T\left|\sin \left(\frac{\theta}{2}\right)\right| \text {. }
$$

By substituting (9) into (7), the static friction force can be determined as

$$
F_{a}=2 \mu T\left|\sin \left(\frac{\theta}{2}\right)\right| \text {. }
$$

Equation (10) shows that static friction in cable-driven mechanism is not constant with respect to joint configuration. To deal with possible asymmetries in the mechanism due to manufacturing imperfection and uncertainties on parameters, such as friction coefficient between cables and bushings, a generalised model for the stiction of a cable driven mechanism can be described by the following equation:

$$
F_{a}=\left\{\begin{array}{ll}
a_{1}+b_{11} T \sin \left(\frac{\theta-c_{1}}{2}\right) & v>0, \theta>c_{1} \\
a_{1}+b_{12} T \sin \left(\frac{\theta-c_{1}}{2}\right) & v>0, \theta<c_{1} \\
a_{2}+b_{21} T \sin \left(\frac{\theta-c_{2}}{2}\right) & v<0, \theta>c_{2} \\
a_{2}+b_{22} T \sin \left(\frac{\theta-c_{2}}{2}\right) & v<0, \theta<c_{2}
\end{array},\right.
$$

where $v$ is the relative velocity between the cable and the bushing, $a_{i}$ is a static force related to the inversion of motion, $b_{i j}$ is the double of the friction coefficient between the cable and the bushing and $c_{i}$ takes into account the manufacturing error.

\section{EXPERIMENTAL VALIDATION}

In this section the hardware prototype used to validate the proposed model, the experiment description and the results discussion are provided.

\section{A. Experimental setup}

The device in Fig. 8 consists of a cable-driven joint operated by a linear actuator able to move two twin cables in opposite directions and a pulley mechanism that aligns the cables with the joint geometry. Besides, Fig. 5 illustrates the cable driven joint used to validate the friction model.

Two force sensors are used to measure the force applied to the joints with a resolution of $5 \mathrm{~N}$. The cable is made of Zylon with a diameter of $3 \mathrm{~mm}$ fixed by splicing the cables around the anchor points in pulling rods and flanges. The actuation system is composed by a Maxon brushless motor coupled to an harmonic gear. The geared motor transfers the motion to the first ball screw, which moves a helical gear mounted on it. The two gears with ratio 1:1 allow the two ball nuts to move with the same speed but in opposite direction. A Renishaw absolute magnetic encoder with a resolution of 18 bits is mounted on the rotation to axis measure the joint angular position with a resolution of $2.4 \times 10^{-5} \mathrm{rad}$.

To run the control algorithm and acquire the measurement signals a National Instrument compactRio is used. A CANbus module has been used for reading the encoders and driving the motor, while force sensors have been acquired through an ADC module with a sample frequency of 250 $\mathrm{kS} / \mathrm{s}$ and 16 bit of resolution.

A PID controller has been implemented to move the cabledriven joint. The coefficients of the friction model proposed in section III have been identified acquiring the cables forces in the whole range of motion of the joint. 




Fig. 9. Actuator friction parameter identification result



Fig. 10. Actuator friction parameter identification result

\section{B. Parameters Identification}

1) Harmonic Gear Friction: To obtain a more realistic simulation, the harmonic gear friction has been modeled as described in [16] as a non linear function of the motor velocity $\omega$ as

$$
\tau_{f}=\alpha \arctan (\beta \omega) .
$$

To identify the $\alpha$ and $\beta$ parameters of (12), the actuator has been driven in velocity mode with no load attached. In this configuration an array of velocity set points has been sent to the motor. For each velocity set point the mean value of the torque and its standard deviation have been computed. Such values have been used to identify the friction model parameters using the non-linear least square algorithm provided by the curve fitting toolbox of Matlab ${ }^{\circledR}$. Fig. 9 shows the fit result and the uncertainty associated to each point. The identified coefficients and their $95 \%$ confidence level are listed in Table II.

2) Cable Stiffness and Internal Damping: Measuring the linear displacement, velocity of the actuator, and the force on the cable $K$ and $c$ parameters can be easily identified through a linear regression. A set of experiments have been carried out connecting one end of the cable rigidly with the chassis and the other end with the linear actuator. In the first part of the experiments the cable has been preloaded up to $200 \mathrm{~N}$ in order to exclude all the non-linear phenomena related to low tension. Then the motor starts to move at a constant low speed ramping the tension up to $500 \mathrm{~N}$.
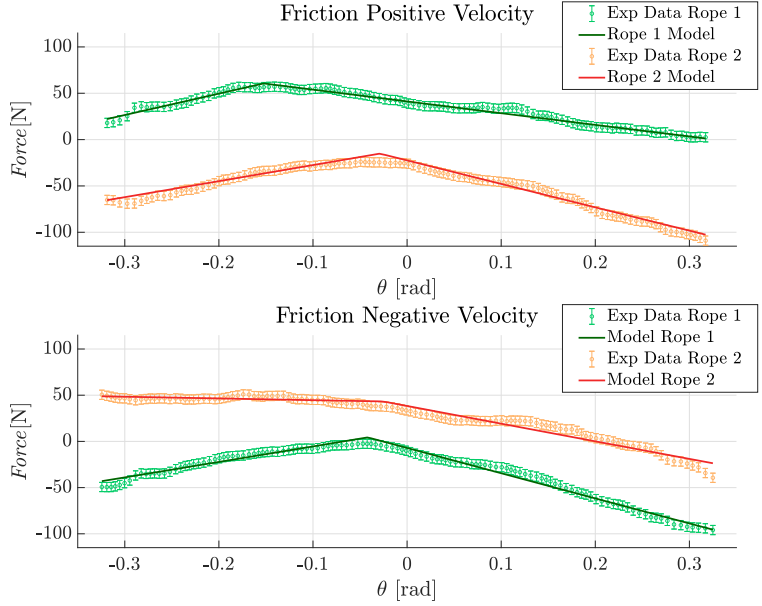

Fig. 11. Cable friction identification results

TABLE II

IDENTIFIED PARAMETERS

\begin{tabular}{|c|c|c|c|}
\hline Coefficient & Estimated Value & Uncertainty & unit \\
\hline \multicolumn{4}{|c|}{ Harmonic Gear Friction Parameters } \\
\hline$\alpha$ & 77.7 & \pm 1.9 & $m N m$ \\
\hline$\beta$ & 0.02 & \pm 0.004 & $s / r a d$ \\
\hline \multicolumn{4}{|c|}{ Cable Parameters } \\
\hline$K$ & 272.1 & \pm 0.2 & $N / m m$ \\
\hline$c$ & 31.6 & \pm 0.7 & $N s / m m$ \\
\hline \multicolumn{4}{|c|}{ Cable 1 Friction Parameters } \\
\hline$a_{1}$ & 60.60 & \pm 0.05 & $N$ \\
\hline$a_{2}$ & 4.35 & \pm 0.05 & $N$ \\
\hline$b_{11}$ & -0.7088 & \pm 0.0011 & \\
\hline$b_{12}$ & 1.283 & \pm 0.005 & \\
\hline$b_{21}$ & -1.5211 & \pm 0.0020 & \\
\hline$b_{22}$ & 0.9346 & \pm 0.0024 & \\
\hline$c_{1}$ & -0.1524 & \pm 0.0003 & rad \\
\hline$c_{2}$ & -0.04185 & \pm 0.00023 & rad \\
\hline \multicolumn{4}{|c|}{ Cable 2 Friction Parameters } \\
\hline$a_{1}$ & -15.11 & \pm 0.06 & $N$ \\
\hline$a_{2}$ & 43.24 & \pm 0.10 & $N$ \\
\hline$b_{11}$ & -1.411 & \pm 0.002 & \\
\hline$b_{12}$ & 0.966 & \pm 0.003 & \\
\hline$b_{21}$ & -1.066 & \pm 0.003 & \\
\hline$b_{22}$ & -0.104 & \pm 0.003 & \\
\hline$c_{1}$ & -0.0289 & \pm 0.0003 & $\operatorname{rad}$ \\
\hline$c_{2}$ & -0.0254 & \pm 0.0007 & $\mathrm{rad}$ \\
\hline
\end{tabular}

The dataset made by the different experiments has been divided into two parts, the former $60 \%$ for identification and the latter $40 \%$ for validation. The result of the estimation is shown in Fig. 10 and the parameters and their 95\% confidence level are listed in Table II.

3) Sliding Cable Friction: The trajectory implemented to estimate the cable sliding friction is made by three setpoints to explore the whole range of motion of the joint under investigation. Every movement is implemented with 


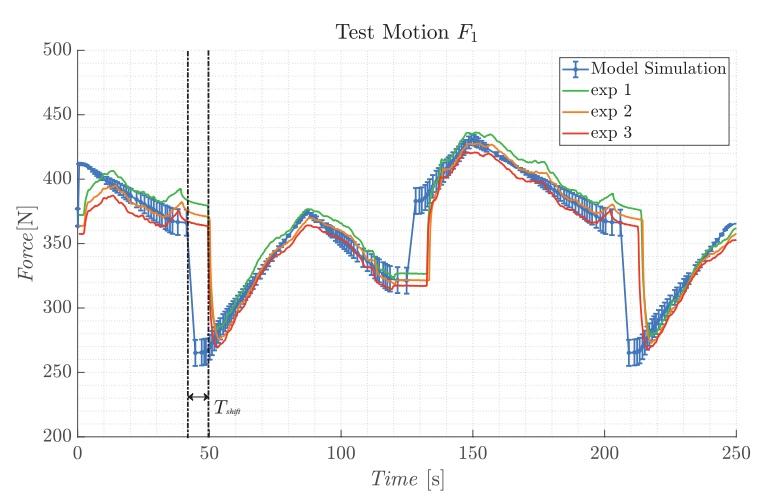

(a)

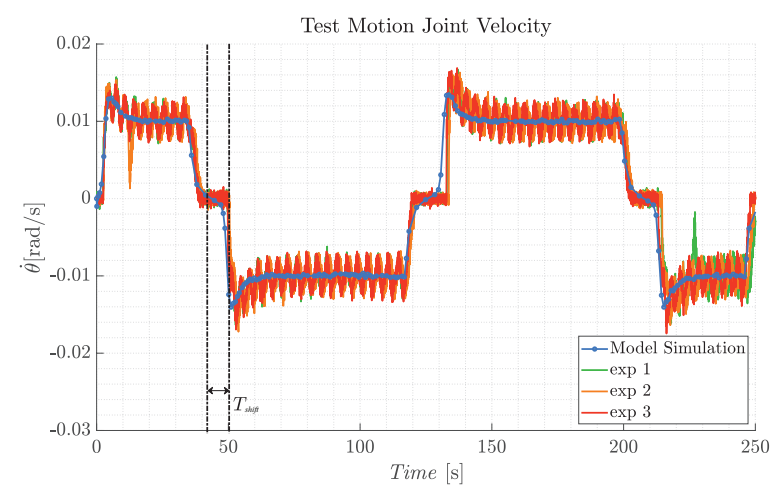

(c)



(b)

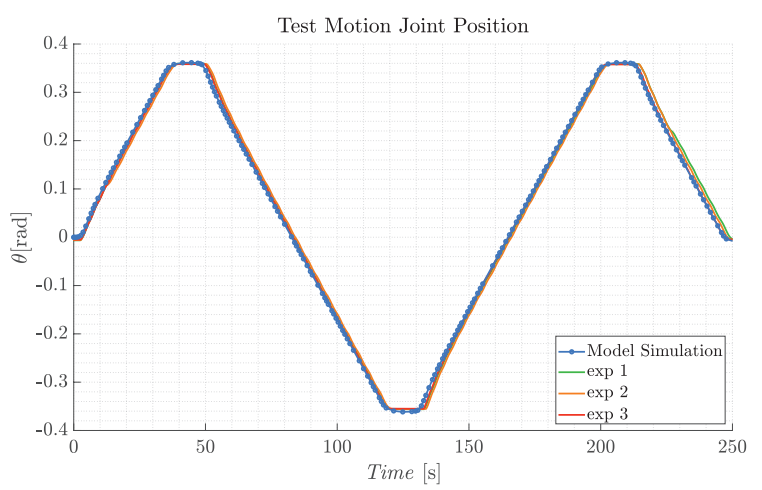

(d)

Fig. 12. Comparison between simulation and experimental data: (a) the forces on the first cable $\left(F_{1}\right)$, (b) the forces on the second cable $\left(F_{2}\right)$, $(c)$ the joint angular velocity $(\dot{\theta})$, and (d) the joint angular position, $(\theta)$.

a trapezoidal velocity profile. During the motion the cable tension force, the angular position of the joint, and the linear velocity of the actuator are recorded. The acquired data are lowpass filtered with a cut-off frequency of $1 \mathrm{~Hz}$ in order to reduce high frequency noise. Then the mean value of the force measured is subtracted to remove the bias induced by the preload of the cable. Finally the non linear fit algorithm has been implemented to find the friction coefficients for both the cables. Fig. 11 shows fit results while the identified parameters and their $95 \%$ confidence level are listed in Table II.

\section{Friction Model Validation}

The model derived in Section II has been implemented in Simulink ${ }^{\circledR}$. Then a simulation of the above described experiment has been performed. Figs. 12a and 12b show the forces measured during the experiment on the first and the second cable respectively and the corresponding forces obtained by the simulation. The joint velocity has been computed by numerically differentiating the joint angular position. Figs. 12c and 12d depict the joint angular velocity and position measured during the experiment and simulation. During the test, the measured velocity plot shows a sinusoidal disturbance, which is caused by the harmonic gear torque ripple not considered in the modeling. As shown by plots Figs. $12 \mathrm{a}$ and $12 \mathrm{~b}$, the forces measured on both the cables are accurately described by the forces computed by the proposed friction model. Furthermore the experimental data lie under the uncertainty bounds obtained from the identification procedure. Figs. 12a and $12 \mathrm{~b}$ present a temporal shift $T_{\text {shift }}$ in force discontinuities. This phenomenon arises because, even if the joint velocity is almost zero, in the simulation appears a change of direction of the motion not present in the real joints. This small difference cause an ill-timed jump in the forces observed. This timing issue is not a problem to introduce this friction model as a feedworward term in a control scheme since the velocity reference is a priori known and does not suffer from error.

\section{CONCLusion}

In this paper the bond-graph approach has been applied to model the dynamics and friction of cable-driven joints. First from the bond-graph formalism the dynamic equations of the system are derived to perform a realistic simulation of the mechanism under test. Subsequently a novel method to model the friction of cables sliding into bushing has been proposed. Then a campaign of experimental tests has been carried out to perform the identification of the parameters of the model proposed. Finally experimental data and simulation results have been compared showing that such model is able to accurately describe the dynamics of a cable-driven joint and the friction associated to cables 
sliding into bushings. In the future work, a feedforward friction compensation is to be implemented to improve the tracking performance of a realtime controller for cable driven mechanisms.

\section{REFERENCES}

[1] N. Simaan, K. Xu, W. Wei, A. Kapoor, P. Kazanzides, R. Taylor, and P. Flint, "Design and Integration of a Telerobotic System for Minimally Invasive Surgery of the Throat," International Journal of Robotics Research, vol. 28, no. 9, pp. 1134-1153, sep 2009.

[2] N. Pedemonte, T. Rasheed, D. Marquez-Gamez, P. Long, É. Hocquard, F. Babin, C. Fouché, G. Caverot, A. Girin, and S. Caro, "Fastkit: A mobile cable-driven parallel robot for logistics," in Advances in Robotics Research: From Lab to Market. Springer, 2020, pp. 141163.

[3] R. O. Buckingham and A. C. Graham, "Link assembly for a snake like robot arm," Jun. 9 2009, uS Patent 7,543,518.

[4] X. Dong, M. Raffles, S. Cobos-Guzman, D. Axinte, and J. Kell, "A Novel Continuum Robot Using Twin-Pivot Compliant Joints: Design, Modeling, and Validation," Journal of Mechanisms and Robotics, vol. 8, no. 2, 11 2015, 021010. [Online]. Available: https://doi.org/10.1115/1.4031340

[5] P. Guardiani, C. Canali, A. Pistone, S. Leggieri, C. Gloriani, N. Rahman, F. Cannella, and D. Caldwell, "Novel integrated robotic system for tiny duct inspection," Procedia Manufacturing, vol. 17, pp. 342 - 349, 2018, 28th International Conference on Flexible Automation and Intelligent Manufacturing (FAIM2018), June 11-14, 2018, Columbus, OH, USAGlobal Integration of Intelligent Manufacturing and Smart Industry for Good of Humanity. [Online]. Available: http://www.sciencedirect.com/science/article/pii/S2351978918311715

[6] L. Gargiulo, P. Bayetti, V. Bruno, J.-C. Hatchressian, C. Hernandez, M. Houry, D. Keller, J.-P. Martins, Y. Measson, Y. Perrot, and F. Samaille, "Operation of an iter relevant inspection robot on tore supra tokamak," Fusion Engineering and Design, vol. 84, no. 2, pp. $220-223,2009$, proceeding of the 25th Symposium on Fusion Technology. [Online]. Available: http://www.sciencedirect.com/science/article/pii/S0920379608003785

[7] R. O. Buckingham and A. C. Graham, "Dexterous manipulators for nuclear inspection and maintenance - case study," in $20101 \mathrm{st}$ International Conference on Applied Robotics for the Power Industry, Oct 2010, pp. 1-6.

[8] G. Endo, A. Horigome, and A. Takata, "Super dragon: A 10-m-longcoupled tendon-driven articulated manipulator," IEEE Robotics and Automation Letters, vol. 4, no. 2, pp. 934-941, April 2019.

[9] A. Marjaninejad, D. Urbina-Meléndez, B. A. Cohn, and F. J. ValeroCuevas, "Autonomous functional movements in a tendon-driven limb via limited experience," Nature machine intelligence, vol. 1, no. 3, pp. 144-154, 2019.

[10] G. P. Bevan, "Bond-graph modeling Complex Embedded Automotive Control Systems (CEmACS) View project Yield factors and AD modelling View project," 2007. [Online]. Available: www.ece.arizona.edu/

[11] N. Mishra and A. Vaz, "Bond graph modeling of a 3-joint string-tube actuated finger prosthesis," Mechanism and Machine Theory, vol. 117, pp. $1-20,2017$. [Online]. Available: http://www.sciencedirect.com/science/article/pii/S0094114X17302495

[12] H. Olsson, K. J. Åström, C. C. De Wit, M. Gäfvert, and P. Lischinsky, "Friction models and friction compensation," Eur. J. Control, vol. 4, no. 3, pp. 176-195, 1998.

[13] M. Miyasaka, J. Matheson, A. Lewis, and B. Hannaford, "Measurement of the cable-pulley Coulomb and viscous friction for a cabledriven surgical robotic system," in IEEE International Conference on Intelligent Robots and Systems, vol. 2015-December. Institute of Electrical and Electronics Engineers Inc., dec 2015, pp. 804-810.

[14] J. B. Coulibaly, M. A. Chanut, S. Lambert, and F. Nicot, "Sliding cable modeling: An attempt at a unified formulation," International Journal of Solids and Structures, vol. 130-131, pp. 1-10, jan 2018.

[15] J. Jung, R. S. Penning, N. J. Ferrier, and M. R. Zinn, "A modeling approach for continuum robotic manipulators: Effects of nonlinear internal device friction," in 2011 IEEE/RSJ International Conference on Intelligent Robots and Systems, Sep. 2011, pp. 5139-5146.

[16] C. Preissner, T. J. Royston, and D. Shu, "A high-fidelity harmonic drive model," Journal of dynamic systems, measurement, and control, vol. 134, no. 1, 2012. 